percentage was calculated 1) using the IMPAX measurement tools 2) by using the 'Hip Screen' phone application and 3) using the radiology report. The three methods were compared.

Results The technical quality of most of the hip radiographs was clinically acceptable. There was a lower percentage of radiographs with adequate pelvic inclination than those with adequate pelvic rotation. There was good agreement between measurements taken with the app, measurements taken directly on IMPAX and the radiology report.

Conclusions Technical quality of the hip radiographs evaluated is generally sufficient to assess hip migration. The 'Hip Screen' phone application can be considered a reliable method of hip surveillance.

Discussion Using the 'Hip Screen' phone application may be more acceptable to future clinicians as may can be readily accessed and may be a useful tool for educating families of children with cerebral palsy.

\section{OC35 PULSE WAVE VELOCITY, FREE FATTY ACIDS, AND STANDARD LIPIDOGRAM IN NORMAL WEIGHT, OVERWEIGHT AND OBESE SCHOOL CHILDREN}

${ }^{1}$ Bernarda Vogrin*, ${ }^{2,3,4}$ Marjan Slak Rupnik, ${ }^{5}$ Dušanka Mičetić-Turk. ${ }^{1}$ PEDENJPED D.0.0., Lenart, Slovenia; ${ }^{2}$ Institute of Physiology, Medical faculty, University of Maribor, Maribor, Slovenia; ${ }^{3}$ Center of physiology and pharmacology, Medical University Vienna, Vienna, Austria; ${ }^{4}$ Alma Mater Europaea - European Center Maribor, Maribor, Slovenia; ${ }^{5}$ Medical faculty, University of Maribor, Maribor, Slovenia

\subsection{6/archdischild-2019-epa.34}

Background and Aim Childhood obesity poses a serious threat for the development of metabolic syndrome, diabetes and cardiovascular diseases (CVD) later in life. Nowdays we are able to recognize metabolic and vascular pathology at a discrete, completely curable stage and long before clinical signs of metabolic syndrome and CVD. Increased arterial stiffness is a good indicator of initial intimal atherosclerotic lesions, easily detected with measuring of pulse wave velocity (PWv) and augmentation index (AIx). Atherosclerosis is initiated and promoted by disturbed lipid metabolism. The increased level of serum free fatty acids (FFA) is supposed to be an early sign of imminent metabolic disturbance. The aim of our study was to assess and compare the metabolic and arterial health in a sample of normal weight, overweight and obese school children from north-west Slovenia.

Materials and Methods 81 healthy, metabolic syndrome free school children aged 11-16 years participated in our study. According to the standard deviation score of body mass index (SDS BMI), the underweight represented $1(1.2 \%)$, the normal weight 38 (46.9\%), the overweight 22 (27.2\%)and the obese $20(24.7 \%)$ of the sample. The AIx, PWv, free fatty acids, standard lipidogram and serum glucose (GLU) were recorded.

Results In the group of overweight children (SDS BMI 1-2) compared to normal weight (SDS BMI -2 to 1) no differences in arterial nor metabolic health parameters were recorded. In the obese children (SDS BMI >2) a significantly hiegher PWv $(\mathrm{p}<0.01)$ triglycerides $(\mathrm{TRG}) \quad(\mathrm{p}<0.01)$, the ratio between TRG and hiegh density lipoproteins (HDL) $(\mathrm{p}<0.001)$, the TRG/low density lipoproteins (LDL) $(p<0.01)$, FFA $(p<0.05)$ and lower HDL $(p<0.01)$ than in normal weight were found.

Conclusion Despite the detected increased arterial stiffness of aorta and signs of metabolic disturbance, the obese children were still free of clinical signs of metabolic syndrome. Our results could be important for planning of preventive and therapeutic meassures of overweight and obese children. According to this study, preventive activities should be provided to the overweight children, to maintain their arterial and metabolic health, while obesity has to be cured promptly to recover the metabolic stability and to cure initial atherosclerotic changes.

\section{OC36 THE UTI-UTI AUDIT: UNDERSTANDING THE TREATMENT OF INFANTS WITH URINARY TRACT INFECTION}

Warren McCue*, Laura Bingham, Lesley-Ann Funston, Shilpa Shah. Craigavon Area Hospital, Craigavon, UK

10.1136/archdischild-2019-epa.35

Background and aims In infants with UTI, practice previously was to commence prophylaxis and investigate for underlying renal pathology. DMSA and MCUG scans have associated risks, and along with urgent inpatient ultrasound, they represent a burden on radiology services. Antibiotic prophylaxis has been linked with rising bacterial resistance.

The current NICE guideline on UTIs in children sets clearer indications for investigation, recommending that only infants with atypical features or recurrence should undergo DMSA and MCUG. Are these recommendations being followed in the youngest infants (0-3 months) diagnosed with UTI?

Methods A retrospective NIECR (Northern Ireland Electronic Care Record) audit was performed on all infants under 3 months diagnosed with UTI in Craigavon Hospital from January - December 2017. Data compared to NICE recommendations and analysed.

Results Data collected for 31 admissions, representing 29 patients: $66 \%$ male, $34 \%$ female.

$55 \%$ classified 'typical', 45\% 'atypical', though $68 \%$ of all cases underwent DMSA/MCUG.

93\% of atypical UTIs were managed as per NICE, compared with only $24 \%$ of typical UTIs. Of the remaining typical cases, 6\% were underinvestigated and 71\% were overinvestigated: $41 \%$ with urgent inpatient US plus DMSA/ MCUG, 30\% with urgent inpatient US alone.

$77 \%$ of atypical cases had significant abnormalities on scans. Only two overinvestigated typical cases, or $17 \%$, showed significant abnormality. However, one had a firstdegree family history of vesicoureteric reflux. This is a recognised NICE atypical feature in older age-groups but is not listed for the $<6$ month group.

Conclusion Atypical UTIs were managed more appropriately than typical UTIs when compared against NICE recommendations. As expected from the evidence base, atypical and recurrent UTIs were commonly associated with underlying renal abnormalities. Typical UTIs, when investigated, were rarely associated with underlying abnormalities or scarring. A laminated aide-mémoire with appropriate investigations is now available in clinical areas on children's ward for quick recall. 